\title{
Probing the Cosmic Star Formation History by Brightness Distribution of Gamma-Ray Bursts
}

\author{
Tomonori Totani \\ Department of Physics, School of Science, The University of Tokyo, Tokyo 113-0033, Japan \\ E-mail: totani@utaphp2.phys.s.u-tokyo.ac.jp
}

\begin{abstract}
Brightness distribution of Gamma-Ray Bursts (GRBs) is studied in detail under the assumption that GRB rate is related to cosmic star formation rate. The two populations of the long- and short-duration bursts in the 4B BATSE catalog are analyzed separately. Taking account of current uncertainties in the observational estimate of star formation rate (SFR), we have tried various models of the cosmic star formation history and we find that star formation history in relatively low redshifts $(z=0-1)$ is strongly constrained by the GRB data. In the Einstein-de Sitter universe with $\left(h, \Omega_{0}, \Omega_{\Lambda}\right)=(0.5,1,0)$, the SFR increase factor from $z=0$ to 1 should be smaller than 3.7 (95\% C.L.) if the rate of the long GRBs is proportional to SFR, and than 5.8 if the long GRBs are produced by binary neutron-star mergers. These values are significantly smaller than the well-known estimate $(\sim 14)$ of the increase factor based on the UV luminosity density, but we argue that this observational value is likely overestimated and the real value may be as low as about 4 if we choose different sets of cosmological parameters and/or take account of the evolution of metallicity and dust extinction. The maximum redshift corresponding to the most faint GRBs observed by the BATSE should be larger than 2, and likely in the range of $3-5$. The beaming factor required for the neutron-star merger scenario is about a few hundreds.
\end{abstract}

Subject headings: binaries: close - stars: neutron-cosmology: observationsgalaxies: evolution-gamma rays: bursts

\section{Introduction}

The brightness distribution of gamma-ray bursts (GRBs) observed by the Burst and Transient Source Experiment (BATSE) has been known to be significantly deficient in faint 
bursts compared with that expected in the Euclidean space (Meegan et al. 1992), and this has been considered as one of the evidences of the cosmological origin of GRBs (Mao \& Paczyński 1992; Piran 1992; Dermer 1992). On the other hand, since most of GRB models are associated to death of massive stars whose lifetime is much shorter than the cosmological time scale, the brightness distribution of GRBs reflects not only the cosmological effects but also the cosmic star formation history (Totani 1997, hereafter T97; Sahu et al. 1997; Wijers et al. 1998). The cosmological origin of GRBs is now confirmed by the discovery of metal absorption lines in the optical counterpart of GRB 970508 (Metzger et al. 1997), and hence more detailed analyses of the GRB brightness distribution are required to investigate the origin of GRBs and the cosmic star formation history.

The most important consequence of the possible relation of GRB rate and star formation rate (SFR) is that the distance scale and absolute luminosity of GRBs become larger than those for no-evolution sources, because we need stronger cosmological effect in order to cancel out the rapid increase of the observed star formation rate from $z=0$ to 1 by a factor of more than 10 (Lilly et al. 1996). However, different authors give quantitatively different results: T97 pointed out that the scenario of binary neutron-star mergers (Blinnikov et al. 1984, referred to "the NS-NS model", hereafter) results in a better fit than the case that GRB rate is simply proportional to SFR (referred to "the proportional model", hereafter), because the time delay during the spiral-in phase of binary neutron stars make the SFR evolution significantly flatter in $z=0-1$. On the other hand, Wijers et al. (1998) concluded that the proportional model is in good agreement with the observed GRB brightness distribution when the redshift of the most distant GRBs is about 6. Petrosian \& Lloyd (1997), however, claimed that neither the NS-NS nor the proportional model is consistent with the observationally determined SFR evolution. This paper investigates the origin of this discrepancy paying special attention to uncertainties in the SFR observations. We found that the GRB rate evolution in $z=0-1$ is crucially important in the GRB distribution analysis, and a stringent constraint on the cosmic star formation history in this redshift range is obtained.

It is well known that the duration of GRBs shows a bimodal distribution suggesting the existence of two populations of long ( $\gtrsim 2 \mathrm{sec}$ ) and short ( $\lesssim 2 \mathrm{sec}$ ) GRBs (Kouveliotou et al. 1993). All previous papers including T97, which investigated the GRB brightness distribution in the context of the cosmic star formation history, however treated the GRBs as a single population. Because the energy spectrum of short GRBs is significantly harder than that of long GRBs (Kouveliotou et al. 1993) and the assumed spectrum affects the GRB brightness distribution analysis, we analyze the GRB distribution separating the two populations in this paper. We define the long GRBs as those with $T_{90}>2$ sec, while the short GRBs with $T_{90}<2$ sec, where $T_{90}$ is the interval over which $5 \%$ to $95 \%$ of the burst 
counts accumulate (Kouveliotou et al. 1993). We also propose a new method to determine the average GRB spectrum in which the curvature of GRB spectrum compared to a pure power-law is taken into account based on the hardness-brightness correlation seen in the BATSE catalog.

The paper is organized as follows: in $\S 2$, formulations used in this paper are described. We determine the average spectrum of the short and long GRBs and give the relations between redshift and peak flux of GRBs. In $\S 3$, the models of cosmic star formation history used in this paper are described. The results of fits to the observed GRB distribution of the 4B BATSE catalog (Paciesas et al. 1997) are given in $§ 4$. After discussing the result and its implications on the cosmic star formation history in $\S 5$, we conclude this paper in $\S 6$.

\section{Formulations}

Expected number of GRBs in a flux interval between $P_{1}$ and $P_{2}\left(P_{1}<P_{2}\right)$, where $P$ is peak photon flux in $\mathrm{cm}^{-2} \mathrm{sec}^{-1}$, is given by

$$
N\left(P_{1}, P_{2}\right)=\zeta T_{\mathrm{obs}} \int_{z\left(P_{2}\right)}^{z\left(P_{1}\right)} \frac{d V}{d z} \frac{R_{\mathrm{GRB}}(z)}{(1+z)} d z
$$

where $d V / d z$ is the comoving volume element per unit redshift, $\zeta$ the sky coverage efficiency [ $38 \%$ for the BATSE experiment (Meegan et al. 1996)], $T_{\text {obs }}$ the observation term, and $R_{\mathrm{GRB}}$ the comoving GRB rate density. The factor $(1+z)^{-1}$ is included to account for the time dilation of the interval between detected bursts. We use the standard candle approximation throughout this paper and hence redshift and peak flux of GRB are related uniquely as $z(P)$. The comoving volume element can be written as $(c=1)$ :

$$
\frac{d V}{d z}=\frac{4 \pi d_{L}^{2}}{(1+z)}\left|\frac{d t}{d z}\right|
$$

where $t$ is the cosmic time and $d t / d z$ in the Freedman universe is given as

$$
\left(\frac{d t}{d z}\right)^{-1}=-H_{0}(1+z) \sqrt{\left(1+\Omega_{0} z\right)(1+z)^{2}-\Omega_{\Lambda}\left(2 z+z^{2}\right)}
$$

and the luminosity distance $d_{L}$ is given as

$$
d_{L}= \begin{cases}\frac{1+z}{H_{0} \sqrt{\Omega_{0}+\Omega_{\Lambda}-1}} \sin \left(d_{\text {prop }} H_{0} \sqrt{\Omega_{0}+\Omega_{\Lambda}-1}\right), & \left(\Omega_{0}+\Omega_{\Lambda}>1\right) \\ (1+z) d_{\text {prop }}, & \left(\Omega_{0}+\Omega_{\Lambda}=1\right) \\ \frac{1+z}{H_{0} \sqrt{1-\Omega_{0}-\Omega_{\Lambda}}} \sinh \left(d_{\text {prop }} H_{0} \sqrt{1-\Omega_{0}-\Omega_{\Lambda}}\right), & \left(\Omega_{0}+\Omega_{\Lambda}<1\right) .\end{cases}
$$


Here the proper distance $d_{\text {prop }}$ is given by

$$
d_{\text {prop }}=\int_{0}^{z}(1+z)\left|\frac{d t}{d z}\right| d z .
$$

The most important fitting parameter in the GRB brightness distribution analysis is the distance scale of GRBs, and in this work we choose $z_{\max }$, the redshift of the most distant bursts corresponding to a threshold flux of the analysis, $P_{\min }$. The $z$ - $P$ relation is determined by the following equation:

$$
\frac{P}{P_{\min }}=\frac{1+z}{1+z_{\max }} \frac{d_{L}^{2}\left(z_{\max }\right)}{d_{L}^{2}(z)} \frac{k_{P}(z)}{k_{P}\left(z_{\max }\right)},
$$

where $k_{P}(z)$ is the $k$-correction for the GRB photon spectrum:

$$
k_{P}(z)=\frac{\int_{(1+z) \varepsilon_{l}}^{(1+z) \varepsilon_{u}} \frac{d L_{n}}{d \varepsilon} d \varepsilon}{\int_{\varepsilon_{l}}^{\varepsilon_{u}} \frac{d L_{n}}{d \varepsilon} d \varepsilon} .
$$

Here $d L_{n} / d \varepsilon$ is the differential photon number luminosity of GRBs at rest, and $\varepsilon$ the photon energy. The energy range in which the peak flux is measured, $\left(\varepsilon_{l}, \varepsilon_{u}\right)$, is $50-300 \mathrm{keV}$ for the BATSE experiment. The factor $(1+z) /\left(1+z_{\max }\right)$ in Eq. 6 is introduced because the standard definition of $d_{L}$ is valid for energy flux but $P$ is photon number flux. The BATSE peak flux is given in three different time scales of 64, 256, and 1024 msec. We use the 1024 msec time scale for the long GRBs because the sensitivity is best in this scale, but 64 msec for the short GRBs because the average duration of the short GRBs is $\sim 0.3$ sec and we have to use shorter time scale than this for the real peak flux. We set the threshold values of $P_{\min }=0.4 \mathrm{~cm}^{-2} \mathrm{sec}^{-1}$ (1024 msec) and $1.6 \mathrm{~cm}^{-2} \mathrm{sec}^{-1}$ (64 msec) for the long and short GRBs, respectively, above which the BATSE trigger efficiency is better than $90 \%$ (Paciesas et al. 1997). There are 773 long GRBs and 192 short GRBs above these thresholds in the 4B catalog.

Now let us determine the photon spectrum of GRBs. Because the spectrum is significantly different for the long- and short-duration bursts, we have to determine the spectra of these populations separately. Figure 1 shows a histogram of power-index of photon spectra $\left(d L_{n} / d \varepsilon \propto \varepsilon^{-\alpha}\right)$ for the long GRBs (dashed line), short GRBs (dot-dashed line), and total (solid line). The values of $\alpha$ are estimated from the ratio of the fluence in 50-100 keV and 100-300 keV in the $4 \mathrm{~B}$ catalog. The average of $\alpha$ for long + short GRBs is 1.38, which is consistent with a recent estimate of $\alpha=1.1 \pm 0.3$ (Mallozzi, Pendleton, \& Paciesas 1996). It is clear from this figure that the long-duration bursts have significantly softer spectra than short bursts; average $\alpha$ is 1.52 and 0.82 for the long and short GRBs, respectively. 
It is an easy option to analyze the GRB distribution with these average photon indices, but here we take a further step to include the effect of curvature of GRB spectra. Figure 2 shows $\alpha-P$ plots for the long and short GRBs. Both populations (especially the long GRBs) show a trend that weaker bursts have softer spectra, and this can be understood as follows. GRB spectra cannot be described by a pure power-law but exhibit some curvature, i.e., the spectrum becomes softer with increasing photon energy (e.g., Mallozzi, Pendleton, \& Paciesas 1996). Because weaker bursts are more distant and hence more redshifted, the observed spectrum becomes softer for weaker GRBs on average. In the following we describe the determination of the GRB spectrum which takes account of this $\alpha-P$ correlation in a consistent way. First we assume a unique relation between $\alpha$ and $P$, which is obtained by a linear fit for the observed $\alpha-P$ relation, as shown in Fig. 2 by the solid lines. We set a maximum peak flux $P_{\max }$, for which we adopt $60 \mathrm{~cm}^{-2} \mathrm{sec}^{-1}$ both for the long and short GRBs, and denote the photon indices corresponding to $P_{\min }$ and $P_{\max }$ as $\alpha_{\text {Pmin }}$ and $\alpha_{\text {Pmax }}$, respectively. We also assume a functional form of $d L_{n} / d \varepsilon$ :

$$
\log \frac{d L_{n}}{d \varepsilon}=\frac{1}{2} A(\log \varepsilon)^{2}+B \log \varepsilon+\text { const. }
$$

Although not physically meaningful, this form describes the four-channel burst spectra of the BATSE GRBs quite well (Mallozzi, Pendleton, \& Paciesas 1996). The two parameters, $A$ and $B$, are determined so that the average photon index in the restframe energy range of $\left[(1+z) \varepsilon_{l},(1+z) \varepsilon_{u}\right]$ is consistent with the $\alpha-P$ relation:

$$
\begin{aligned}
& A\langle\log \varepsilon\rangle_{P_{\max }}+B=-\alpha_{\mathrm{Pmax}} \\
& A\langle\log \varepsilon\rangle_{P_{\min }}+B=-\alpha_{\mathrm{Pmin}}
\end{aligned}
$$

where

$$
\begin{aligned}
\langle\log \varepsilon\rangle_{P_{\max }} & =\frac{1}{2}\left\{\log \left[\left(1+z_{\min }\right) \varepsilon_{l}\right]+\log \left[\left(1+z_{\min }\right) \varepsilon_{u}\right]\right\} \\
\langle\log \varepsilon\rangle_{P_{\min }} & =\frac{1}{2}\left\{\log \left[\left(1+z_{\max }\right) \varepsilon_{l}\right]+\log \left[\left(1+z_{\max }\right) \varepsilon_{u}\right]\right\} .
\end{aligned}
$$

The above spectrum is determined if $z_{\max }$ and $z_{\min }$, which are the redshifts corresponding to $P_{\min }$ and $P_{\max }$, are fixed. The fitting parameter $z_{\max }$ can be chosen arbitrary and the unknown parameter $z_{\text {min }}$ is determined by solving the following consistency equation:

$$
\frac{P_{\max }}{P_{\min }}=\frac{1+z_{\min }}{1+z_{\max }} \frac{d_{L}^{2}\left(z_{\max }\right)}{d_{L}^{2}\left(z_{\min }\right)} \frac{k_{P}\left(z_{\min }\right)}{k_{P}\left(z_{\max }\right)} .
$$

Now the GRB spectrum, which depends on the fitting parameter $z_{\text {max }}$, has been determined. We note that the above treatment of the GRB spectrum is the first to take account of 
the $\alpha-P$ correlation in a consistent way. In Figure 3, we show some examples of the GRB spectrum determined by the above method. The spectral curvature becomes larger with decreasing $z_{\max }$ because the restframe spectrum has to become softer more rapidly with increasing photon energy.

Now the relation between $z$ and $P$ is fixed, and the $z-P$ plots are shown in Fig. [4 both for the long and short GRBs for some values of $z_{\max }$. Since the $z$-P relation has been fixed, we can calculate the cosmological time dilation factor and redshift of some particular GRBs as functions of $z_{\max }$. Cosmological time dilation effect has been searched for various temporal structures, and a dilation factor of about 2 for the long duration bursts is widely accepted (see, e.g., Norris et al. 1994, 1995; Fenimore \& Bloom 1995). Recently Che et al. (1997) suggested the existence of time dilation of about 2 also for the short GRBs. In the upper panel of Fig. 5, we plot the time dilation factor, $\left(1+z_{\text {weak }}\right) /\left(1+z_{\text {bright }}\right)$, as a function of $z_{\max }$. The brighter and weaker peak fluxes are taken as $\left(P_{\text {weak }}, P_{\text {bright }}\right)=(0.46$, $9.5)$ and $(1.6,10.0)$ for the long and short GRBs, respectively. The values for the long GRBs correspond to the "dim+dimmest" and "bright" populations defined by Norris et al. (1994, see also Horack, Mallozzi, \& Koshut 1996 for estimate of peak flux for these groups), and those for the short GRBs roughly correspond to the minimum and maximum of the brightness parameter defined by Che et al. (1997). The dilation factor of about 2 suggests $z_{\max } \sim 2$ both for the long and short GRBs. The lower panel of this figure shows the redshift of GRB970508, which is a long GRB with $1024 \mathrm{msec}$ peak flux of $0.85 \mathrm{~cm}^{-2} \mathrm{sec}^{-1}$ (when $\alpha=1.5$, see Kouveliotou et al. 1997), as a function of $z_{\max }$. The constraint of $0.835 \leq z<2.3$ for this GRB (Metzger et al. 1997) suggests $1.22 \leq z_{\max }<3.43$, although possible dispersion in GRB luminosity might relax this constraint.

Although we have chosen the maximum redshift as the distance parameter of GRBs, it would be convenient to give relations between $z_{\max }$ and absolute luminosity or total emitted energy of GRBs. If the gamma-ray emission is isotropic, the absolute peak luminosity in the restframe energy range of $\left(\varepsilon_{l}, \varepsilon_{u}\right)$ is given as:

$$
L_{\text {peak }}=\langle\varepsilon\rangle \frac{4 \pi d_{L}\left(z_{\max }\right)^{2} P_{\min }}{k_{P}\left(z_{\max }\right)\left(1+z_{\max }\right)},
$$

where $\langle\varepsilon\rangle$ is the average energy in restframe energy range of $\left(\varepsilon_{l}, \varepsilon_{u}\right)$. The total energy emitted by GRBs in the same energy range can be estimated from $f_{\min }$, which is the fluence of GRBs at $z=z_{\max }$ :

$$
E_{\text {tot }}=\frac{4 \pi d_{L}\left(z_{\max }\right)^{2} f_{\min }}{k_{e}\left(z_{\max }\right)\left(1+z_{\max }\right)},
$$


where $k_{e}(z)$ is the $k$-correction for the energy spectrum,

$$
k_{e}(z)=\frac{\int_{(1+z) \varepsilon_{l}}^{(1+z) \varepsilon_{u}} \varepsilon \frac{d L_{n}}{d \varepsilon} d \varepsilon}{\int_{\varepsilon_{l}}^{\varepsilon_{u}} \varepsilon \frac{d L_{n}}{d \varepsilon} d \varepsilon} .
$$

We have estimated $f_{\min }$ from fluence- $P$ plots for the BATSE catalog. The peak luminosity and total energy emitted in the restframe energy range of 50-300 keV are shown in Fig. 6 as functions of $z_{\max }$, as well as the values after bolometric correction (50-2000 keV). Since the spectrum becomes harder, the bolometric correction factor becomes larger with increasing $z_{\max }$. It should be noted that the bolometric correction is based on a simple extrapolation of the assumed functional form of $d L_{n} / d \varepsilon$, but there might be cut-off energy in the GRB spectrum in reality. Therefore the estimate of bolometric luminosity or total energy has to be taken with care, especially for the short GRBs.

\section{Modeling the Cosmic GRB Rate History}

We can now calculate the GRB brightness distribution and compare it to the observation, provided that $R_{\mathrm{GRB}}$ is determined as function of $z$. In this paper we investigate two possibilities: the proportional model and the NS-NS model. Figure 7 shows observational estimations of comoving SFR density based on $\mathrm{H} \alpha$ or UV luminosity density (Gallego et al. 1995; Lilly et al. 1996; Connolly et al. 1997; Madau, Pozzetti, \& Dickinson 1998). In the Einstein-de Sitter universe with $\left(\Omega_{0}, \Omega_{\Lambda}\right)=(1,0)$, the cosmic SFR at $z=1$ is about 15 times higher than the local value, and the data at higher redshifts suggest a peak of cosmic SFR at $z \sim 1-2$. Let $\xi(z)$ be the ratio of SFR at $z$ to the local SFR, i.e., $\xi(z)=R_{*}(z) / R_{*}(0)$, where $R_{*}$ is the comoving SFR density in the universe in $\left[M_{\odot} \mathrm{yr}^{-1} \mathrm{Mpc}^{-3}\right]$. In this paper, we parametrize the cosmic star formation history by two parameters of $\xi(1)$ and $\xi(4)$, and SFR evolution is determined by the natural cubic spline interpolation (e.g., Press et al. 1992) between SFRs at $z=0,1$, and 4 . From the observational data, we adopt $\xi(1)=13.6$ and $\xi(4)=3.43$ as the standard observational star formation history (solid line in Fig. (7).

Although the UV luminosity density evolution in $z=0-1$ measured by the CanadaFrance Redshift Survey (CFRS; Lilly et al. 1996) is very steep, another estimate of this evolution favors more modest evolution. Totani, Yoshii, \& Sato (1997; hereafter TYS) pointed out that the present-day colors of galaxies are strongly correlated to recent star formation history in them and it is possible to estimate the SFR evolution at $z \lesssim 1$ from stellar population synthesis models of galaxies. The estimate of TYS for $\xi(1)$ is about 4 if the age of the universe is reasonable (10-15 Gyr). This value is significantly lower than the 
result of Lilly et al. (1996), but if one assumes a $\Lambda$-dominated universe with $\left(\Omega_{0}, \Omega_{\Lambda}\right)=$ $(0.2,0.8)$ the UV-estimated $\xi(1)$ becomes smaller by a factor of 1.8 and the discrepancy is a little remedied. Furthermore, Hammer et al. (1997) pointed out another effect which may be responsible for the very high $\xi(1)$ in the CFRS; star forming galaxies in the CFRS at $z>0.5$ seem to have significantly low metallicities and hence they may be more transparent to UV light than local galaxies. We will later discuss these points after we obtain the constraints on $\xi(1)$ from the GRB distribution.

It is also widely discussed that the UV-estimated SFR at $z>1$ is highly uncertain and may be significantly underestimated because of the dust extinction effect (Meurer et al. 1997; Pettini et al. 1997; Sawicki \& Yee 1998; Heckman 1998; Cimatti et al. 1998). The ratio of far infrared luminosity to UV in metal-rich $\left(\sim Z_{\odot}\right)$ local starburst galaxies is $10-100$ (Heckman 1998) and this suggests that high redshift galaxies at $z>2$, which also show signatures of starbursts, might be significantly affected by interstellar dust extinction by factor of $\sim 10$ (Meurer et al. 1997; Sawicki \& Yee 1998) or in some cases $\sim 500$ (Cimatti et al. 1998). This possible upward correction for high redshift SFR data is interesting because the formation of spheroidal systems such as elliptical galaxies or bulges of spiral galaxies also predicts higher SFR at $z \gtrsim 2$, if they were formed by starbursts at high redshifts. In order to give a quantitative estimate, let us calculate the total amount of star formation in elliptical galaxies. The total B-band luminosity density at present is $1.2 \times 10^{8} h L_{B} \odot$ $\mathrm{Mpc}^{-3}$ (Lilly et al. 1996), where $h=H_{0} /\left(100 \mathrm{~km} \mathrm{~s}^{-1} \mathrm{Mpc}^{-1}\right)$, with the fraction of elliptical galaxies of 0.28 (Totani, Yoshii, \& Sato 1997). By using the mass-to-luminosity ratio $(M / L)_{B}=16.2 h$ for elliptical galaxies (Faber \& Gallagher 1979), the total amount of stars which have been formed in elliptical galaxies becomes $7.6 \times 10^{8} h^{2}\left[M_{\odot} \mathrm{Mpc}^{-3}\right]$. Here we have taken account of a recycling factor of 1.4 inferred from a galaxy evolution model of Arimoto \& Yoshii (1987). The horizontal dotted lines in Fig. 7 are the average SFR expected in elliptical galaxies if they were formed before $z=2$ or 3 . This result suggests that the upward correction by a factor of $\sim 10$ is very likely from the viewpoint of spheroidal system formation. Considering the uncertainties discussed above, we will try various values of $\xi(1)$ and $\xi(4)$ in the following GRB analysis. We have plotted some examples of the model SFR evolution with different values of $\xi(1)(=13.6$ or 4$)$ and $\xi(4)(=3.43$ or 34.3$)$ in Fig. 7.

If GRBs are associated to NS-NS mergers, we cannot use the simple approximation of $R_{\mathrm{GRB}} \propto R_{*}$, and the GRB rate evolution is written as:

$$
R_{\mathrm{GRB}}(t)=\int_{0}^{t} P_{m}\left(t-t^{\prime}\right) R_{*}\left(t^{\prime}\right) d t^{\prime},
$$

where $t$ is the cosmic time and $P_{m}(x)$ is the probability distribution of time delay from star formation to NS-NS mergers per unit mass of star formation, in units of $\left[M_{\odot}^{-1} \mathrm{yr}^{-1}\right]$. 
The form of $P_{m}$ can be well approximated as $P_{m}(x)=A\left(x / t_{c}\right)^{\gamma}$ with $t_{c} \sim 0.02$ Gyr and $\gamma \sim-1$ (T97). More detailed calculations of $P_{m}$ by binary population synthesis suggest the uncertainty in $\gamma$ is probably about 0.2-0.3 (Lipunov et al. 1997; Portegies Zwart and Yungelson 1998). Because the aim of this paper is to investigate the cosmic star formation history from GRBs, we fix the parameters $t_{c}$ and $\gamma$ to 0.02 Gyr and -1 , respectively. The normalization factor, $A$, can be inferred also from binary population synthesis calculations. Lipunov et al. (1995) estimated the present merger rate as $2 \times 10^{-4}$ $\left[\mathrm{yr}^{-1}\right.$, when $10^{11} M_{\odot}$ stars have been formed constantly. Portegies Zwart \& Yungelson (1998), by similar calculations, found the present rate as $3.4 \times 10^{-5} \mathrm{yr}^{-1}$, when the SFR is constant at $4 M_{\odot} \mathrm{yr}^{-1}$ and the present age of the Galaxy is $10 \mathrm{Gyr}$. Requiring these values to be consistent with the form of $P_{m}$, we find $A=1.6 \times 10^{-13}$ and $7 \times 10^{-14}\left[M_{\odot}^{-1} \mathrm{yr}^{-1}\right]$ for the results of the two groups, respectively. We use $A=1 \times 10^{-13}\left[M_{\odot}^{-1} \mathrm{yr}^{-1}\right]$ for calculation of the absolute rate of NS-NS mergers and the uncertainty in $A$ is probably about a factor of 2 , as suggested from the difference of the two group. In Fig. 8 we show some examples of calculation of $R_{\mathrm{GRB}}(z)$, generated from the four models of $R_{*}(z)$ shown in Fig. 7 . The GRB rate evolution becomes flatter than the star formation history when we take account of the time delay, and the importance of this effect is clear if one compares these results to those neglecting the delay (dotted lines).

\section{Comparison to the BATSE Data}

\subsection{Brightness Distribution of GRBs}

T97 used the Kolmogorov-Smirnov test for the comparison between the observation and the model calculation, which utilizes the cumulative flux distribution, $N(>P)$. However, the differential flux distribution, $d N / d P$, reflects the GRB rate evolution better than $N(>P)$ and we use the $\chi^{2}$ analysis for the differential distribution in this paper. We set 9 bins in $\log P$ with a constant interval both for the long and short bursts.

Adopting the standard observational star formation history, i.e., $\xi(1)=13.6$ and $\xi(4)=3.43$, we have calculated the expected number of GRBs in each bin with nine values of $z_{\max }$ (1-5 with an interval of 0.5 ), and the results are shown in the upper panels of Fig. 9 for the long bursts. (We have chosen the best-fit normalizations for the model calculations and hence only the shape of GRB flux distribution is tested.) In the upper-left panel the GRB rate is assumed to be proportional to SFR while the upper-right panel assumes the NS-NS merger scenario. These panels show that the inclination of the model curves is hardly changed by $z_{\text {max }}$ in peak flux larger than $\sim 10 \mathrm{~cm}^{-2} \mathrm{sec}^{-1}$, while in small $P$ range the expected event number becomes significantly smaller with increasing $z_{\max }$, because of 
the paucity of SFR at high $z$ in the observational star formation history. The standard observational SFR evolution does not agree with the GRB distribution, in either the proportional or NS-NS model. Because the inclination in the bright range of $P$ is almost insensitive to $z_{\max }$, we can set a stringent constraint on the SFR evolution at relatively low redshift $(z \lesssim 1)$. Actually these results suggest that the SFR evolution inferred from the UV luminosity density at $z<1$ (Lilly et al. 1996) is too steep to be consistent with the GRB brightness distribution, both in the proportional model and the NS-NS model. The only way to get a better fit is to decrease the value of $\xi(1)$. In the lower panels, a lower value of $\xi(1)=4$, as inferred from the galaxy evolution model (TYS), is used and then the discrepancy in the bright $P$ range is significantly remedied. Figure 10 is the same with Fig. 9 but for the short GRB population. Although the statistics is poor, the UV-estimated SFR evolution in $z \sim 0-1$ is too steep also for the short GRBs if the GRB rate is proportional to SFR.

The serious discrepancy between the UV-estimated SFR evolution in $z=0-1$ and GRB distribution can also be seen in a SFR- $z$ diagram. When GRB rate is proportional to SFR, we can convert the observed number of GRBs directly into cosmic SFR. Let $N_{\text {obs }}\left(P_{1}, P_{2}\right)$ be the observed number of GRBs in the interval of $P_{1}<P<P_{2}$. Then average SFR in the redshift interval of $z\left(P_{2}\right)<z<z\left(P_{1}\right)$ can be written as:

$$
R_{*} \propto \frac{N_{\mathrm{obs}}\left(P_{1}, P_{2}\right)}{\int_{z\left(P_{2}\right)}^{z\left(P_{1}\right)} \frac{d V}{d z} \frac{d z}{(1+z)}} .
$$

Figure 11 shows evolution of cosmic SFR calculated from the observed number of long GRBs as a function of $z$ with various $z_{\max }$, where the binning in $P$ is the same with that in Fig. 9. These curves are normalized to the observed local SFR at minimum redshifts. The SFR evolution inferred from GRBs becomes steeper with increasing $z_{\max }$ but the evolution in $z=0-1$ is much flatter than the UV-estimated evolution even when we take an extreme value of $z_{\max }=10$. Therefore we conclude that the UV-estimated SFR evolution is inconsistent with the hypothesis that brightness distribution of GRBs traces the cosmic star formation history. In order to get an acceptable fit, one must reduce the high SFR at $z=1$, and this is consistent with the previous result of T97 who found that the star formation history based on the galaxy evolution model of TYS (i.e., $\xi(1) \sim 4$ ) gives a good fit but the observational SFR evolution does not. This result is also consistent with that obtained by Petrosian \& Lloyd (1997), but does not agree with that of Wijers et al. (1998). 


\subsection{Constraints on Cosmic SFR Evolution}

In this section we give quantitative constraints on the cosmic SFR evolution, i.e., $\xi(1)$ and $\xi(4)$, by detailed statistical comparison of the theoretical and observed brightness distribution. Figure 12 shows the allowed regions of $\xi(1)$ and $\xi(4)$ for the long and short GRBs, both for the proportional model and the NS-NS model. In the $\chi^{2}$ test, we have searched the best-fit $z_{\max }$ for each set of $(\xi(1), \xi(4))$, and depicted the contours of exclusion confidence levels (CLs) with the best-fit $z_{\max }$. Therefore in the excluded region there is no acceptable fit with any value of $z_{\max }$. As discussed in the previous section, the most important result is the constraint on $\xi(1)$ : for the long GRBs, $\xi(1)<3.7$ (proportional) and $\xi(1)<5.8(\mathrm{NS}-\mathrm{NS})$ at $95 \%$ CL., for any values of $\xi(4)$ and $z_{\max }$. The time delay in the NS-NS scenario makes the GRB rate evolution flatter in $z=0-1$ and hence allows acceptable fits with larger $\xi(1)$ than the proportional model. The $\xi(1)$ is constrained also for the short GRBs in the proportional model: $\xi(1)<6.48(95 \% \mathrm{CL})$. If the SFR at $z=1$ is more than 10 times higher than the present rate as suggested by the UV luminosity density, the proportional model is completely rejected both for the long and short GRBs, and the NS-NS model is also inconsistent with the distribution of the long GRBs. In order to see the effect of changing the cosmological parameters, we show the same contour maps in Fig. 13 for the long GRBs in the two different cosmological models: open $\left[\left(h, \Omega_{0}, \Omega_{\Lambda}\right)=(0.6,0.2,0)\right]$ and lambda $\left[\left(h, \Omega_{0}, \Omega_{\Lambda}\right)=(0.7,0.2,0.8)\right]$. The constraint on $\xi(1)$ becomes weaker in the open universe, but the upper limit for $\xi(1)$ in the proportional model is still much smaller than 10 .

Figure 14 shows allowed regions in the $\left(z_{\max }, \xi(4)\right)$ space for the long GRBs with a fixed value of $\xi(1)=4$, with which there exist acceptable fits (thick contours). The NS-NS model gives a better fit than the proportional model because of the flatter evolution due to the time delay, and for the proportional model the allowed region exists only in the open universe. The best-fit normalization parameters, i.e., production rate of GRBs per unit mass of star formation (events $/ M_{\odot}$ ) for the proportional model and the beaming factor $(4 \pi / \Delta \Omega)$ for the NS-NS model, are shown by the thin contours in the same figure. The production rate of GRBs required for the proportional model is $\sim 5 \times 10^{-8}$ events $/ M_{\odot}$ if the emission is isotropic, and the NS-NS model requires a beaming factor of $\sim 200-400$. Figure 15 is the same as Figure 14, but for the case of $\xi(1)=3$. If the SFR evolution in $z=0-1$ is as modest as this level, the proportional model becomes consistent with the BATSE data in any cosmological model. The maximum redshift is at least larger than 2 and likely larger than 3, depending on $\xi(4)$. If the distance scale of GRBs is determined independently, the cosmic SFR at high redshift $(z \sim 4)$ can be estimated. 


\section{3. $\quad$ Long and Short GRBs}

Because of the poor statistics of the short GRBs, we cannot derive strong constraints on them except for the upper limit on $\xi(1)$ in the proportional model. However, it may be interesting to see whether there is a difference between the distance scale of the long and short GRBs, provided that the two populations are originated by the same physical event, i.e., the GRB rate evolution of the two populations as a function of $z$ is the same. In Figure 16 we plotted the reduced $\chi^{2}$ of the fit as a function of $z_{\max }$ for the long and short GRBs in the NS-NS model, using a fixed star formation history $[(\xi(1), \xi(4))=(4,34.3)]$. This result suggests that $z_{\max }$ of the long GRBs is larger than that of the short GRBs, and combined with the result of Fig. 6, the total energy emitted by the short GRBs in the restframe 50-300 keV is more than one order of magnitude smaller than that by the long GRBs if the beaming factor of these two populations is the same.

\section{Discussion}

The upper limits on $\xi(1)$ obtained in this paper are quite stringent and obviously inconsistent with the popular value of $\xi(1)>10$ estimated by UV luminosity, in either case of the proportional model or the NS-NS model. However, as we discuss below, it is premature to abandon these models but it is rather likely that the real SFR evolution is flatter than inferred from the UV evolution. The most important uncertainty in UV-estimated SFR is the effect of dust extinction. Hammer et al. (1997, hereafter H97) pointed out that CFRS galaxies at $z \sim 1$ show signatures of low metallicity and smaller dust extinction, which may cause an overestimate of SFR evolution in $z=0-1$. H97 made

a simple model to include this effect, in which the conversion factor between SFR and [OII] luminosity depends on the restframe $(U-V)$ color of galaxies and the dependence on the color is determined by SFR calibration of local galaxies. Then they found that the SFR evolution is $\xi(1) \sim 6.9$ from the CFRS data in the Einstein-de Sitter universe, which is considerably lower than the pure evolution of $2800 \AA$ or $[\mathrm{O}$ II] comoving luminosity density $(\xi(1) \sim 15)$. If we change the cosmological model into the open $\left[\left(\Omega_{0}, \Omega_{\Lambda}\right)=(0.2,0)\right]$ or the $\Lambda$-dominated universe $\left[\left(\Omega_{0}, \Omega_{\Lambda}\right)=(0.2,0.8)\right]$, the value of UV-estimated $\xi(1)$ is further reduced to $\xi(1)=5.33$ and 3.81 , respectively. These values are now near the independent estimate $(\xi(1) \sim 4)$ based on a galaxy evolution model which reproduces the present-day properties of galaxies (Totani, Yoshii, \& Sato 1997). Therefore we consider that the stringent upper limits on $\xi(1)$ from the GRB distribution do not reject the GRB models associated to death of massive stars, but rather require a significant downward correction for the SFR evolution in $z=0-1$ based on UV luminosity. 


\section{Summary and Conclusions}

In this paper we have presented a detailed study on the possible relation between the brightness distribution of gamma-ray bursts and the cosmic star formation history. The long and short GRBs in the 4B BATSE catalog are analyzed separately. We proposed a new method to determine the average GRB spectrum in which the curvature of GRB spectra compared to a pure power-law is taken into account based on the observed $\alpha-P$ correlation. Various models of the cosmic star formation history are tried considering the present uncertainties in the observational estimate of SFRs, and implications of the GRB distribution on the star formation history and galaxy evolution are discussed.

We show that the evolution of SFR in $z=0-1$ is crucially important for the fit of GRB brightness distribution, and star formation history up to $z \sim 1$ is strongly constrained by the GRB data. The analysis on the long GRBs suggests that, in the Einstein-de Sitter universe with $\left(h, \Omega_{0}, \Omega_{\Lambda}\right)=(0.5,1,0)$, the SFR increase factor from $z=0$ to $1[\xi(1)]$ should be smaller than 3.7 (95\% C.L.) if the GRB rate is proportional to SFR, and than 5.8 if the GRBs are produced by binary neutron-star mergers. If the rate of the short GRBs is proportional to SFR, it is also required that $\xi(1)$ is smaller than 6.5 (95 \% C.L.). These values are significantly smaller than the current estimate $(\xi(1) \sim 15$; Lilly et al. 1996) of SFR evolution based on the UV luminosity density, but consistent with a theoretical estimate $(\xi(1) \sim 4)$ by a galaxy evolution model based on the local properties of galaxies (Totani, Yoshii, \& Sato 1997). This result is consistent with Petrosian \& Lloyd (1997), and also with Totani (1997) in which the star formation history based on the galaxy evolution model gave a good fit while the observational history did not. We have argued that the uncomfortably large $\xi(1)$ inferred from the UV observation is likely an overestimation and the real value could be as low as $\sim 4$ if we choose different sets of cosmological parameters and/or take account of the evolution of metallicity and dust extinction. Therefore the UV observation itself is also consistent with the BATSE data and the galaxy evolution model. SFR at high redshifts ( $\gtrsim 2$ ) cannot be strongly constrained, but it requires better determination of the distance scale of GRBs $\left(z_{\max }\right)$.

Although we have explored various models of cosmic SFR evolution, we could not find any acceptable fit for the long GRBs with $z_{\max }<2$. Therefore we conclude that $z_{\max }$ is at least larger than 2 and likely in the range of $z_{\max }=3-5$, if the long GRBs are associated to death of massive stars or NS-NS mergers. The maximum redshift of the short GRBs seems smaller than that of the long GRBs if the GRB rate evolution is the same for the two populations. This suggests that the total energy emitted by the short bursts in the restframe $50-300 \mathrm{keV}$ is smaller than that by the long bursts by more than one order of magnitudes (see Fig. 6), if the beaming factor is the same. The production rate of GRBs 
from star formation is $\sim 5 \times 10^{-8}\left[M_{\odot}^{-1}\right]$ if GRB rate is proportional to SFR, and the beaming factor required for the NS-NS merger scenario is about a few hundreds.

The author has been supported by the Research Fellowships of the Japan Society for the Promotion of Science for Young Scientists, and the Grant-in-Aid for the Scientific Research Fund (No. 3730) of the Ministry of Education, Science, and Culture of Japan.

\section{REFERENCES}

Arimoto, N. \& Yoshii, Y. 1987, A\&A, 173, 23

Blinnikov, S. I. et al. 1984, Sov. Astr. Lett. 10, 177

Che, H., Yang, Y., Wu, M., \& Li, Q.B. 1997, ApJ, 483, L25

Cimatti, A., Andreani, P., Röttgering, H., \& Tilanus, R. 1998, Nature 392, 895

Connolly, A. J. et al. 1997, ApJ, 486, L11

Dermer, C. D. 1992, Phys. Rev. Lett., 68, 1799

Faber, S. M. \& Gallagher, J. S. 1979, ARA\&A, 17, 135

Fenimore, E.E. and Bloom, J.S. 1995, ApJ, 453, 25

Gallego, J., Zamorano, J., Aragon-Salamanca, A., \& Rego, M. 1995, ApJ, 455, L1 (erratum: 459, L43)

Hammer, F. et al. 1997, ApJ, 481, 49 (H97)

Heckman, T.M. 1998, To appear in the proceedings of 'The Most Distant Radio Galaxies', ed. D. Reidel (astro-ph/9801155)

Horack, J. M., Mallozzi, R. S., \& Koshut, T. M. 1996, ApJ, 466, 21

Kouveliotou, C. et al. 1993, ApJ, 413, L101

Kouveliotou, C. et al. 1997, IAU Circ. 6660

Lilly, S. J., Fèvre, O. Le., Hammer, F., \& Crampton, D. 1996, ApJ, 460, L1

Lipunov, V. M. et al. 1995, ApJ, 454, 593

Madau, P., Pozzetti, L., \& Dickinson, M. 1998, ApJ, 498, 106 
Mallozzi, R. S., Pendleton, G. N., \& Paciesas, W. S. 1996, ApJ, 471, 636

Mao, S. \& Paczyński, B. 1992, ApJ, 388, L45

Meegan, C. A. et al. 1992, Nature, 355, 143

Meegan, C. A. et al. 1996, ApJS, 106, 65

Metzger, M.R. et al. 1997, Nature, 387, 878

Meurer, G.R. et al. 1997, AJ, 114, 54

Norris, J.P. et al. 1994, ApJ, 424, 540

Norris, J.P. et al. 1995, ApJ, 439, 542

Paciesas, W. S. et al. 1997, 4B BATSE catalog is available at http://cossc.gsfc.nasa.gov/cossc/BATSE.htm

Petrosian, V. \& Lloyd, N.M. 1997, To appear in the proceedings of the 4th Huntsville Gamma-Ray Burst Symposium, eds. C.A.Meegan, P.Cushman (astro-ph/9711193)

Pettini, M. et al. 1997, to appear in 'The Ultraviolet Universe at Low and High Redshift', ed. W. Waller, (Woodbury: AIP Press), astro-ph/9708117

Piran, T. 1992, ApJ, 389, L45

Portegies Zwart, S.F. \& Yungelson L.R. 1998, A\&A, 332, 173

Press, W.H. et al. 1992, Numerical Recipes, 2nd edition (Cambridge: Cambridge Univ. Press)

Sahu, K. et al. 1997, ApJ, 489, L127

Sawicki, M \& Yee, H.K.C. 1998, AJ, in press astro-ph/9712216

Totani, T. 1997, ApJ, 486, L71 (T97)

Totani, T., Yoshii, Y., \& Sato, K. 1997, ApJ, 483, L75 (TYS)

Wijers, R.M.J., Bloom, J.S., Bagla, J.S. \& Natarajan, P. 1998, MNRAS, 294, L17 


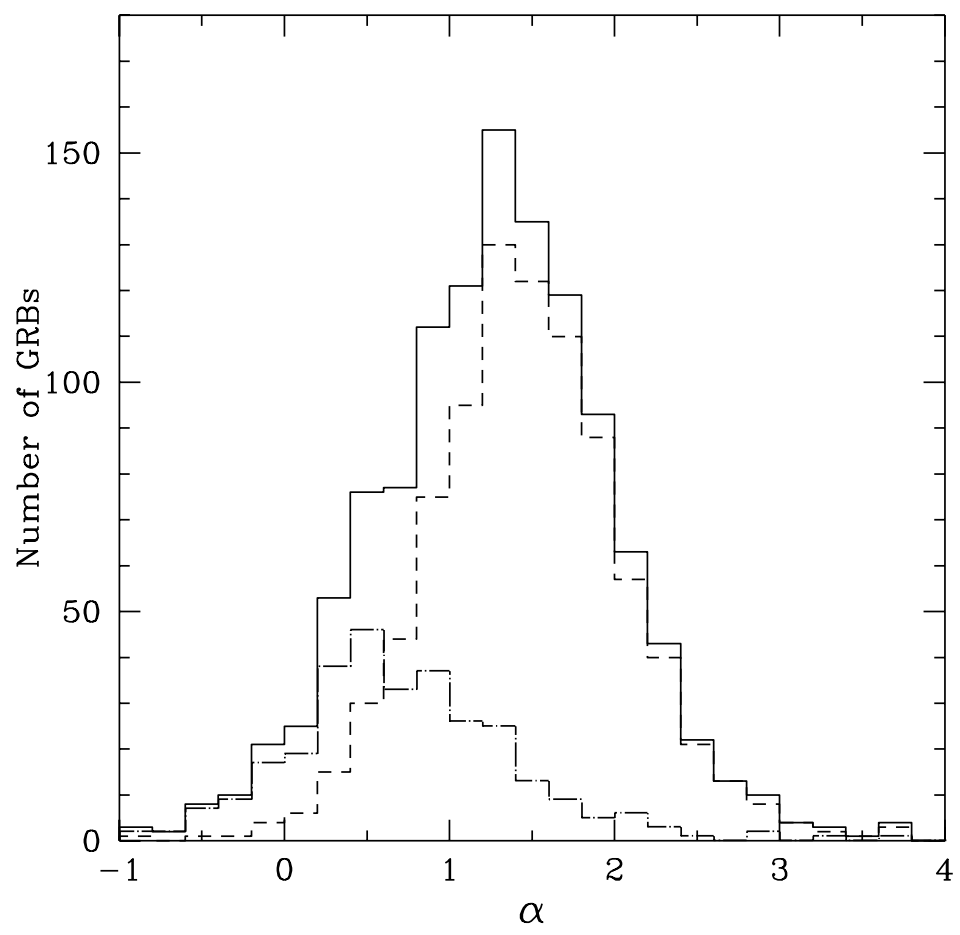

Fig. 1.- The histogram of effective photon index of GRB spectra $(\alpha)$ in the 4B BATSE catalog (Paciesas et al. 1997). The photon index is calculated from the fluence ratio of the 50-100 keV and 100-300 keV ranges. The dashed line is for the long GRBs, the dot-dashed line for short GRBs, and the solid line for the total. 

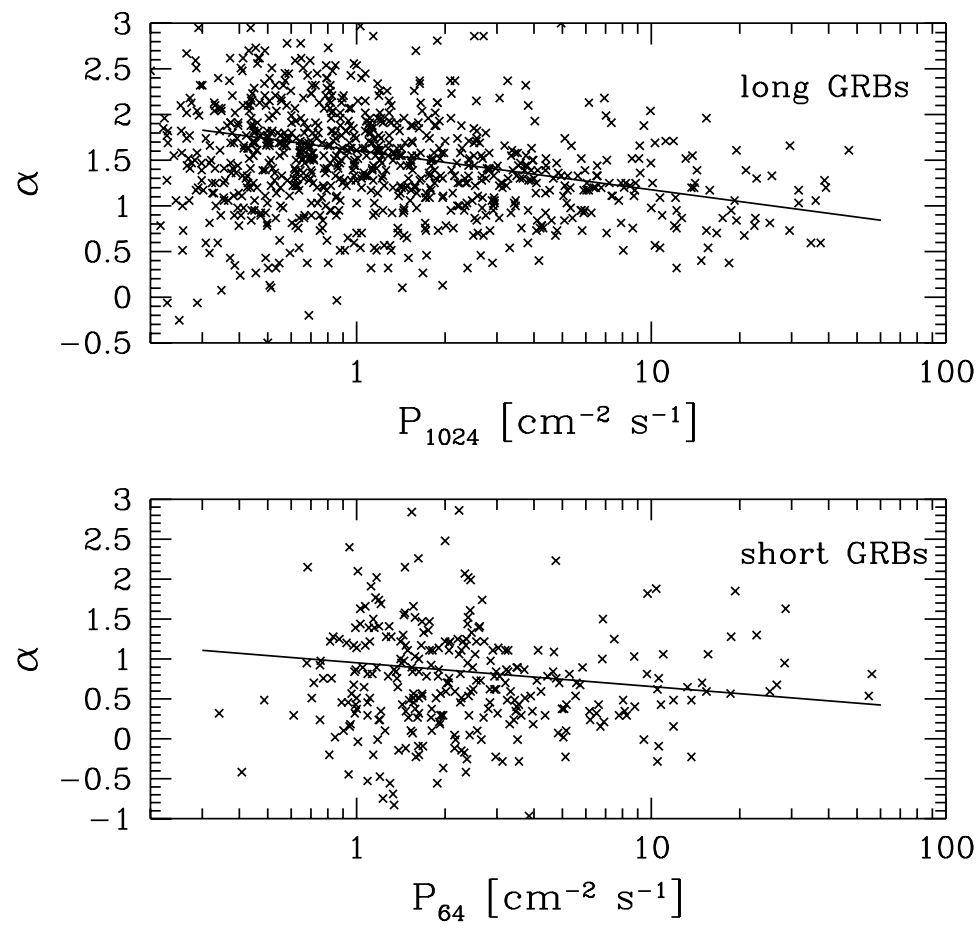

Fig. 2.- Correlation between the photon index $(\alpha)$ and peak flux (1024 msec for the long GRBs and $64 \mathrm{msec}$ for the short GRBs), in the 4B BATSE catalog (Paciesas et al. 1997). The solid lines are least-square fits. 


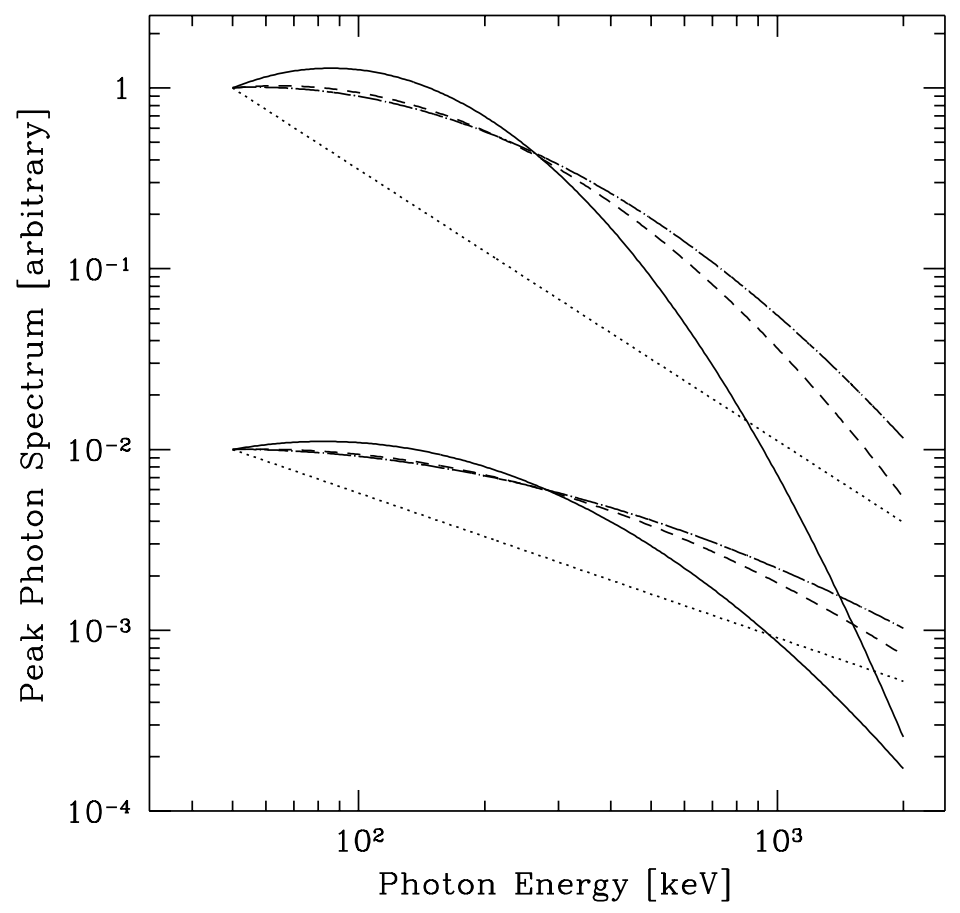

Fig. 3.- The photon spectrum of GRBs used in this paper. In our treatment, the spectrum is determined when the fitting parameter, $z_{\max }$ is fixed (see text). The curvature reflects the $\alpha-P$ correlation shown in Fig. 2. The upper three curves correspond to the long GRBs and the lower to the short GRBs. The solid, dashed, and dot-dashed lines are for the cases of $z_{\max }=1,3$, and 5 , respectively. The dotted lines are simple power-law spectra with the average photon indices of the 4B catalog $(\alpha=1.52$ and 0.82 for the long and short GRBs, respectively). 


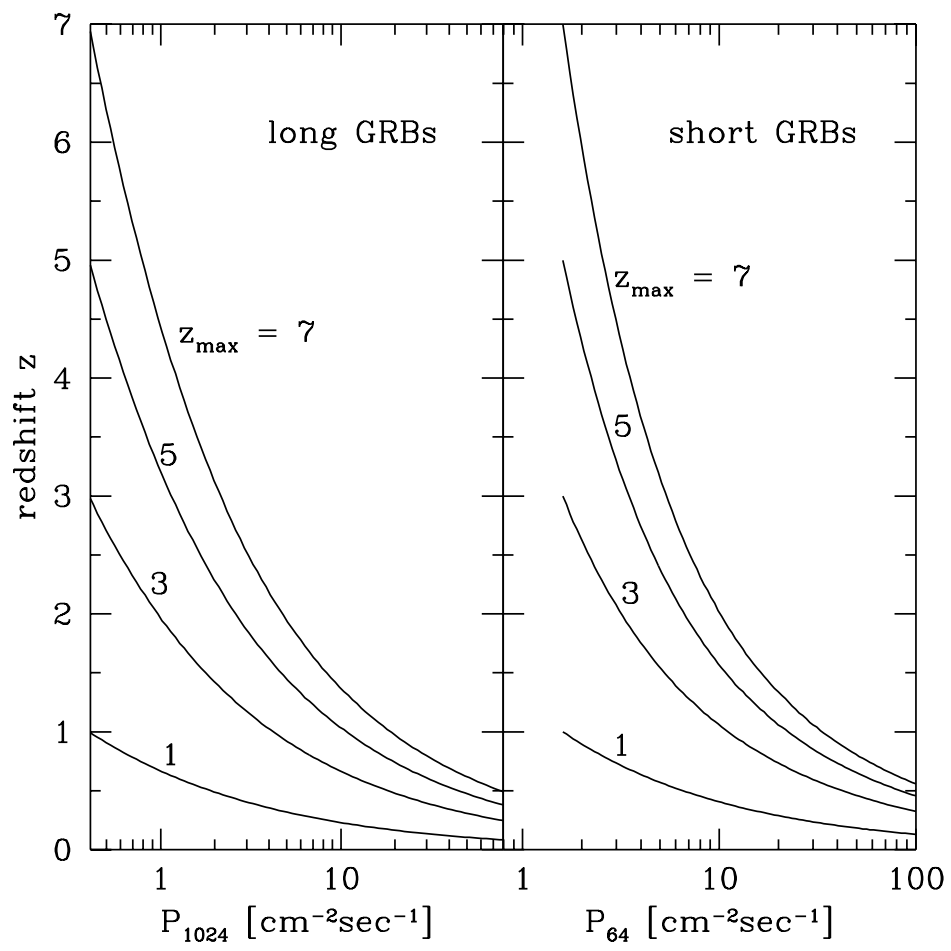

Fig. 4.- The redshift vs. peak flux relation for the long and short GRBs, for some values of $z_{\max }$ which is the redshift corresponding to $P_{1024}=0.4$ and $P_{64}=1.6 \mathrm{~cm}^{-2} \mathrm{sec}^{-1}$ for the long and short GRBs. The values are indicated in the figure. 


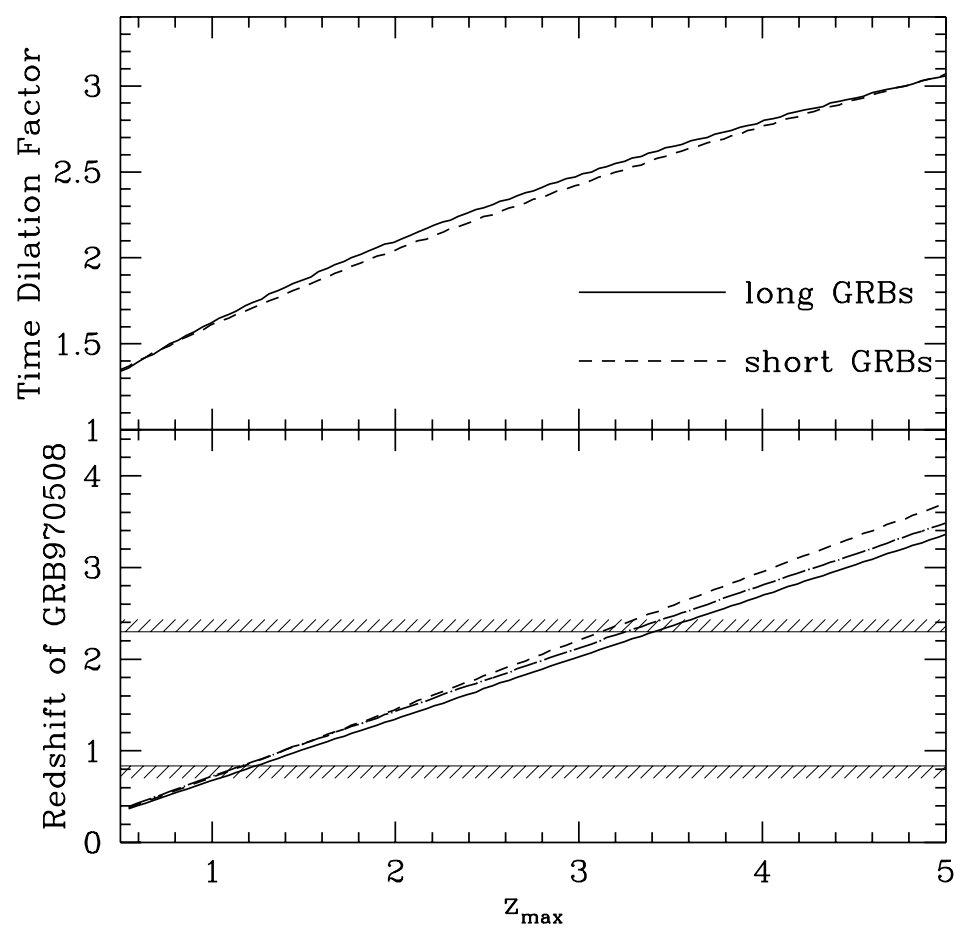

Fig. 5.- Time dilation factor and redshift of GRB 970508, as functions of $z_{\max }$. For the definition of time dilation factor, see text. In the upper panel, the solid line is for the long GRBs and the dashed line for the short GRBs. In the lower panel, the three lines are all for the long GRBs with different cosmological parameters: $\left(h, \Omega_{0}, \Omega_{\Lambda}\right)=(0.5,1,0),(0.6,0.2,0)$, and $(0.7,0.2,0.8)$ for the solid, dashed, and dot-dashed lines, respectively. The constraint of $0.835 \leq z<2.3$ which is set by the absorption lines (Metzger et al. 1997) is also shown. 


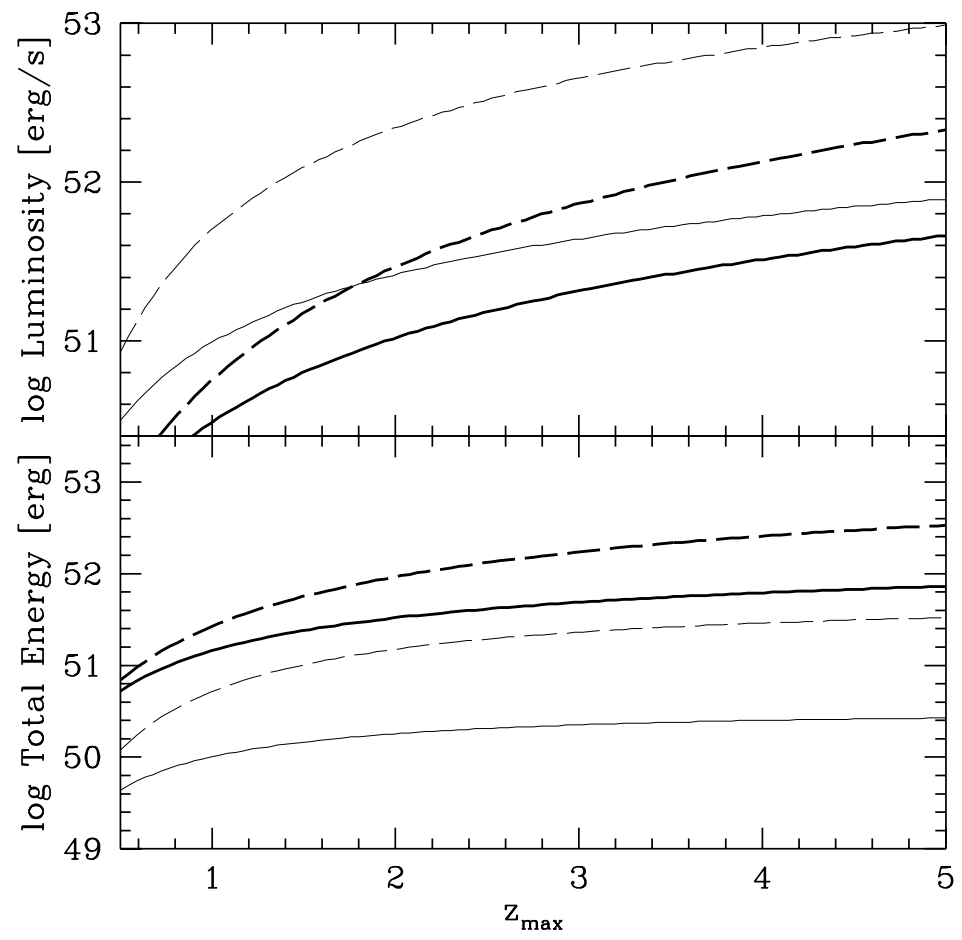

Fig. 6.- Peak luminosity and total energy emitted by GRBs, as functions of the fitting parameter, $z_{\max }$. The thick lines are for the long GRBs, in the energy range of 50-300 keV (solid) and 50-2000 keV (dashed) at the rest frame, while the thin lines are for the short GRBs. Isotropic emission is assumed in this figure. 


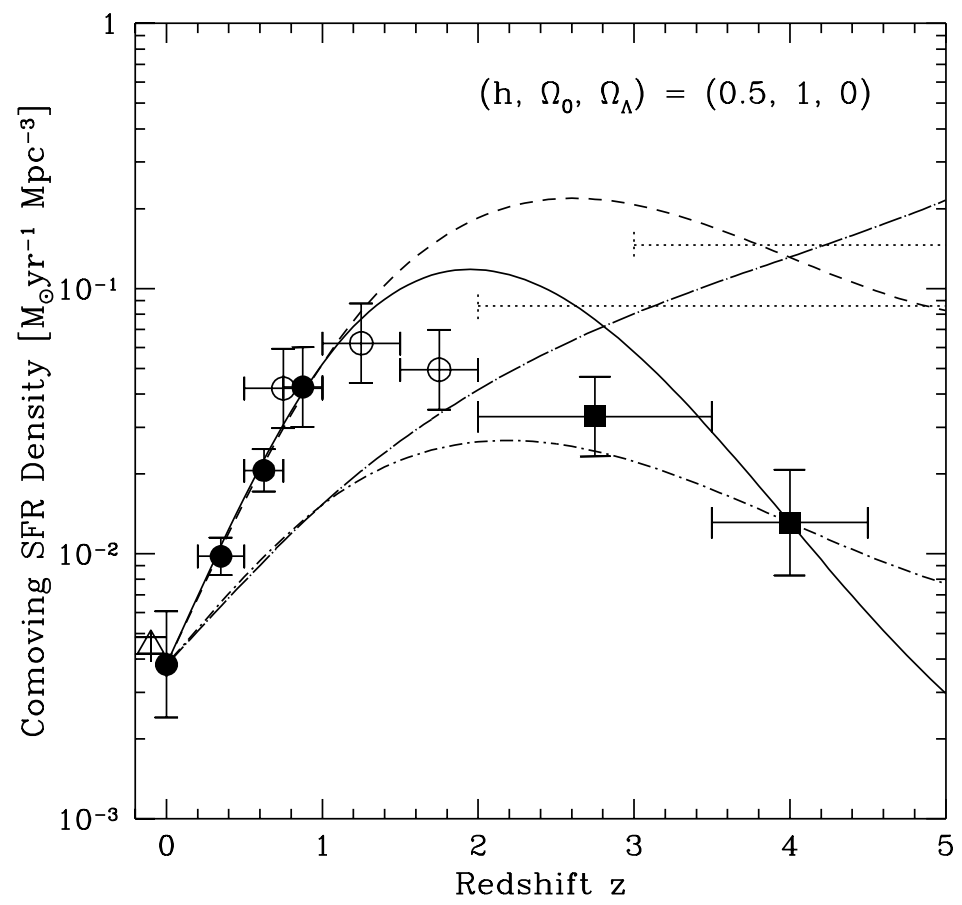

Fig. 7.- Cosmic star formation history as a function of redshift. Data points are direct observational estimates by Gallego et al. (1995, open triangle), Lilly et al. (1996, filled circles), Connolly et al. (1997, open circles), and Madau et al. (1997, filled square). Curves are the model of cosmic star formation history used in this paper, with the parameters $(\xi(1), \xi(4))=(13.6,3.43),(13.6,34.3),(4,3.43)$, and $(4,34.3)$ for the solid, dashed, shortdot-dashed, and long-dot-dashed lines, respectively (see text in detail). The two horizontal dotted lines are average star formation rates expected if the stars in present-day elliptical galaxies were formed before $z=2$ or 3 . 


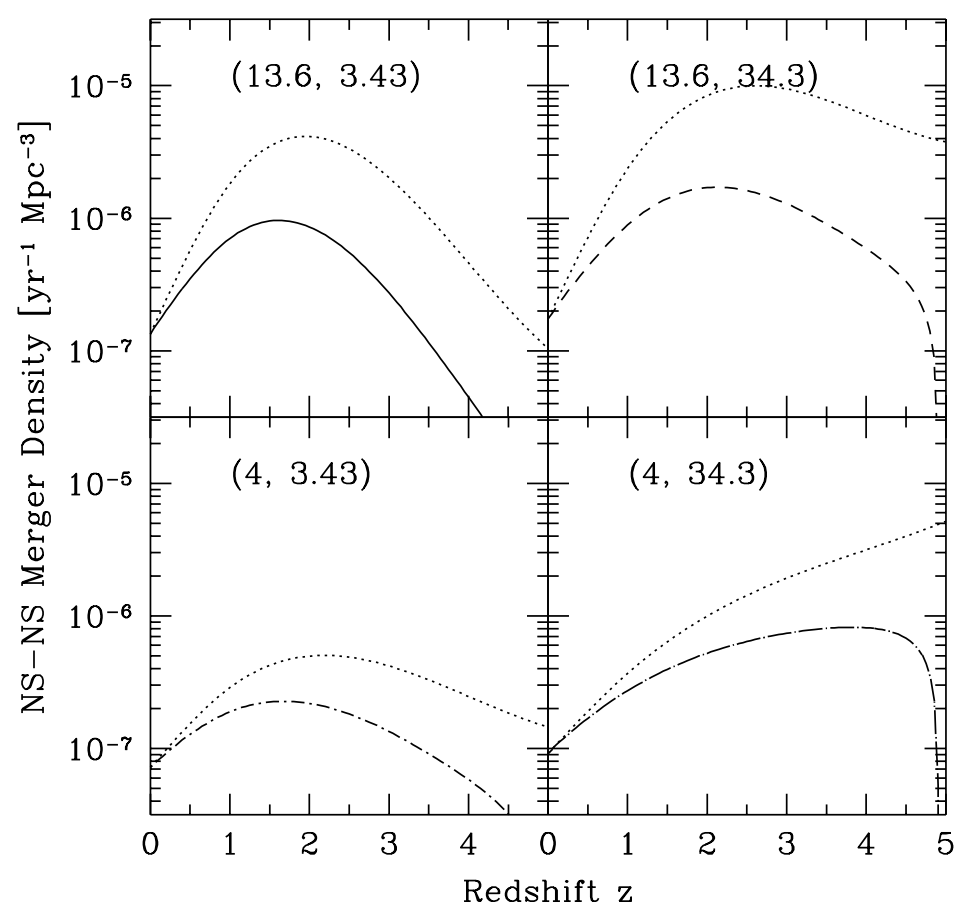

Fig. 8.- The merger rate evolution of binary neutron stars as a function of redshift, calculated from the cosmic SFR evolution shown in Fig. 7. The four panels correspond to the different four models of cosmic SFR evolution with values of $(\xi(1), \xi(4))$ indicated in the figure. The line marking corresponds to that of Fig. 7. The dotted lines are the SFR evolution before convolved with the time delay, normalized at $z=0$ for comparison. The time delay between star formation and merger makes the rate evolution flatter in $z=0-1$. 


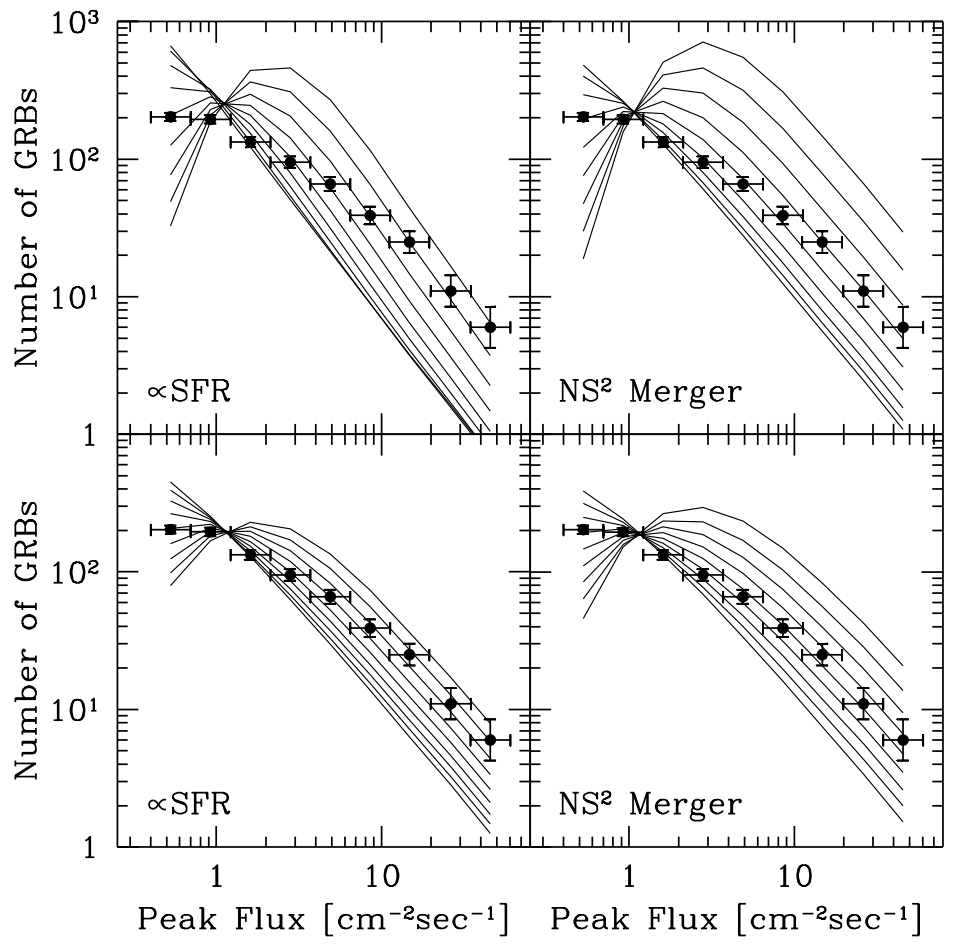

Fig. 9.- Differential distribution of peak flux (1024 msec) of the long GRBs. The data points are the BATSE data and the error bars in peak flux indicate the width of bins. Nine model curves are shown for each panel, corresponding to different values of $z_{\max }=1-5$ with an interval of 0.5. The number of GRBs at small peak flux decreases with increasing $z_{\max }$ because of the low SFR at high redshifts. The left panels are for the proportional model, while the right panels for the NS-NS model. In the upper panels we use $\xi(1)=13.6$ while in the lower panels $\xi(1)=4$. In all panels $\xi(4)=3.43$. 


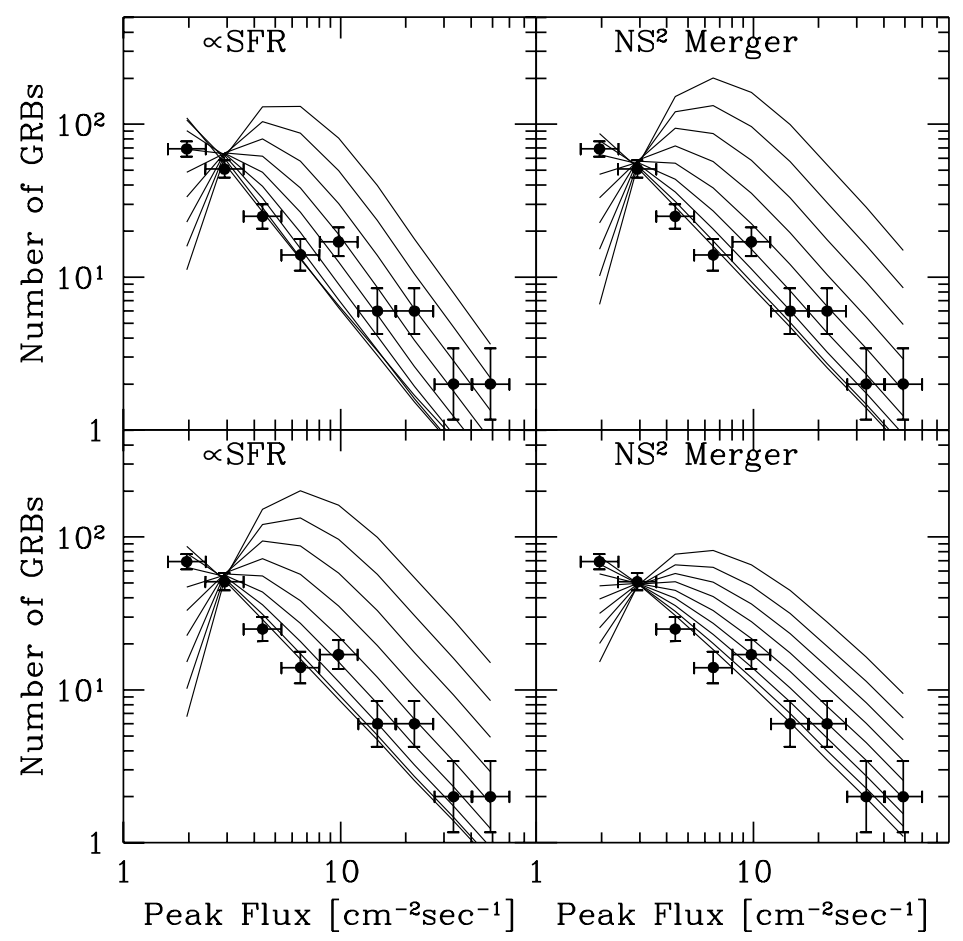

Fig. 10.- The same as Fig. 9, but for the short GRBs with 64 msec peak flux. 


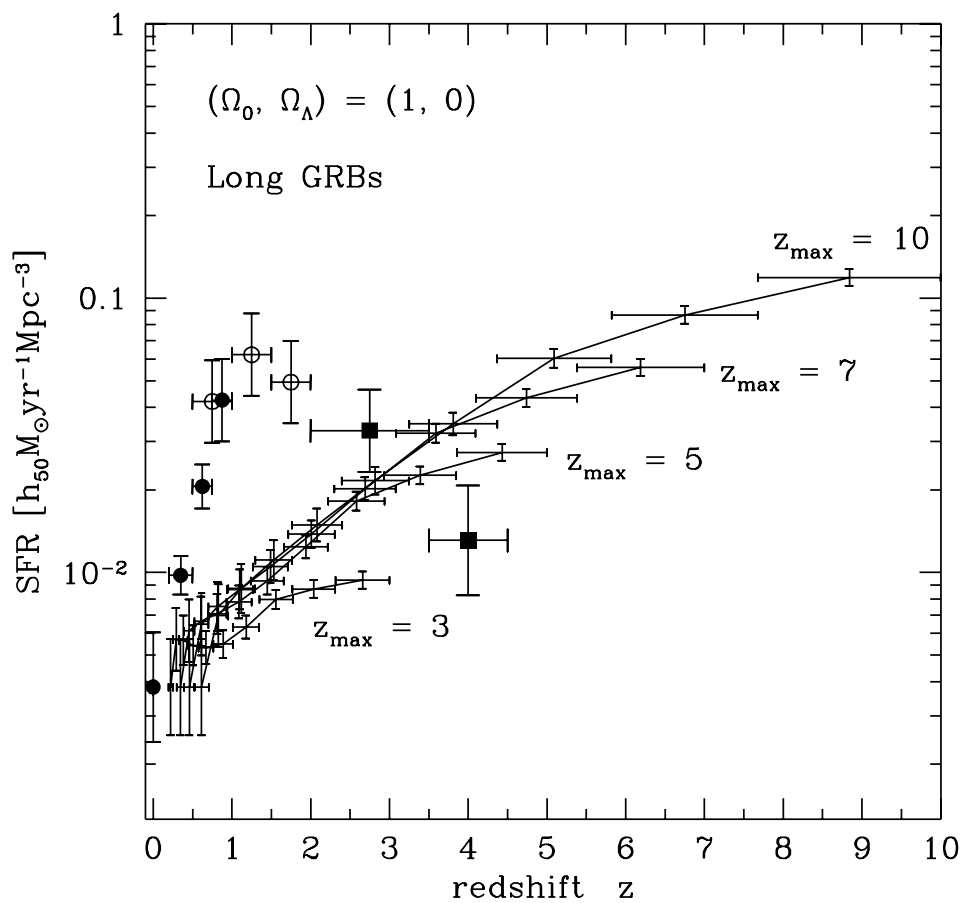

Fig. 11. - The star formation history in the universe deduced from the brightness distribution of the long GRBs (crosses combined with solid lines). The GRB rate is assumed to be proportional to SFR. Four different values of $z_{\max }$ are used, as indicated in the figure. The data points of filled and open circles, and filled squares are the observational SFR estimates as shown in Fig. 7 . 


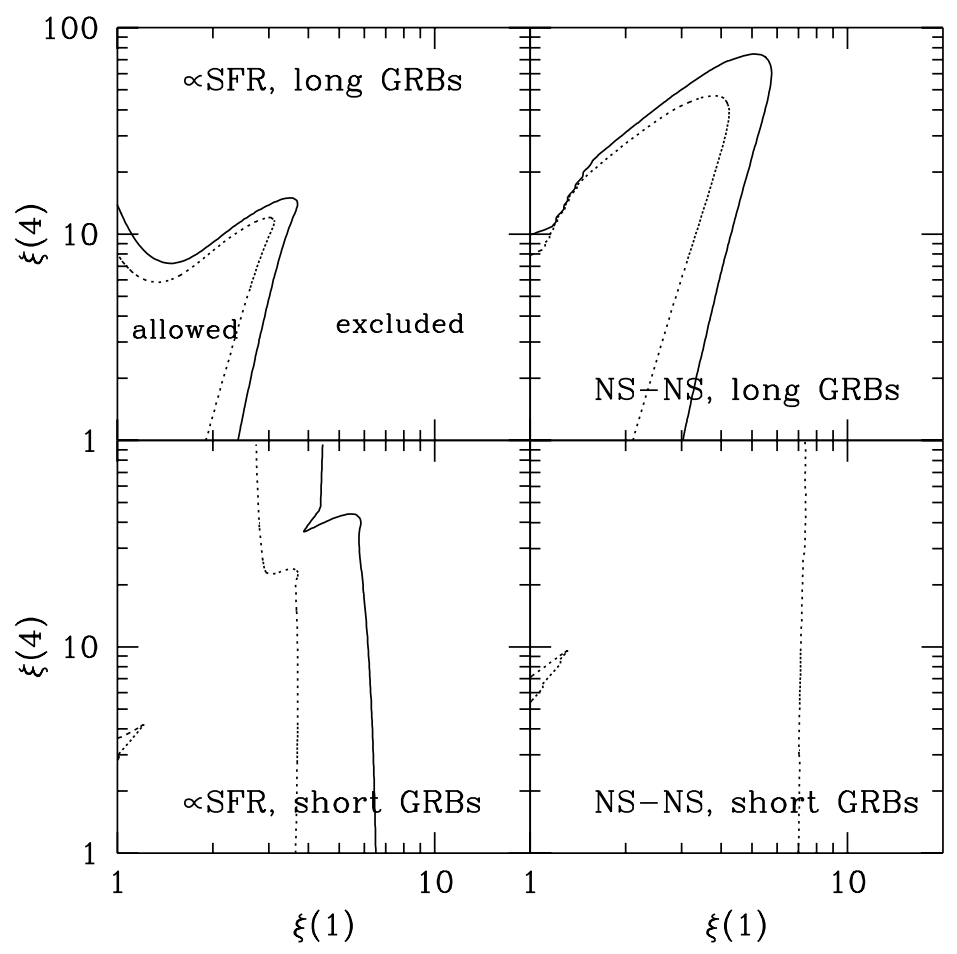

Fig. 12.- Constraints on the cosmic star formation history from GRB brightness distribution. The allowed regions of $\xi(1)$ and $\xi(4)$ are shown for the long and short GRBs (upper and lower panels, respectively) and for the proportional model and the NS-NS model (left and right panels, respectively). The solid line shows the $95 \%$ C.L. constraints, while the dotted lines for $68 \%$ C.L. The Einstein-de Sitter universe with $h=0.5$ is assumed. 


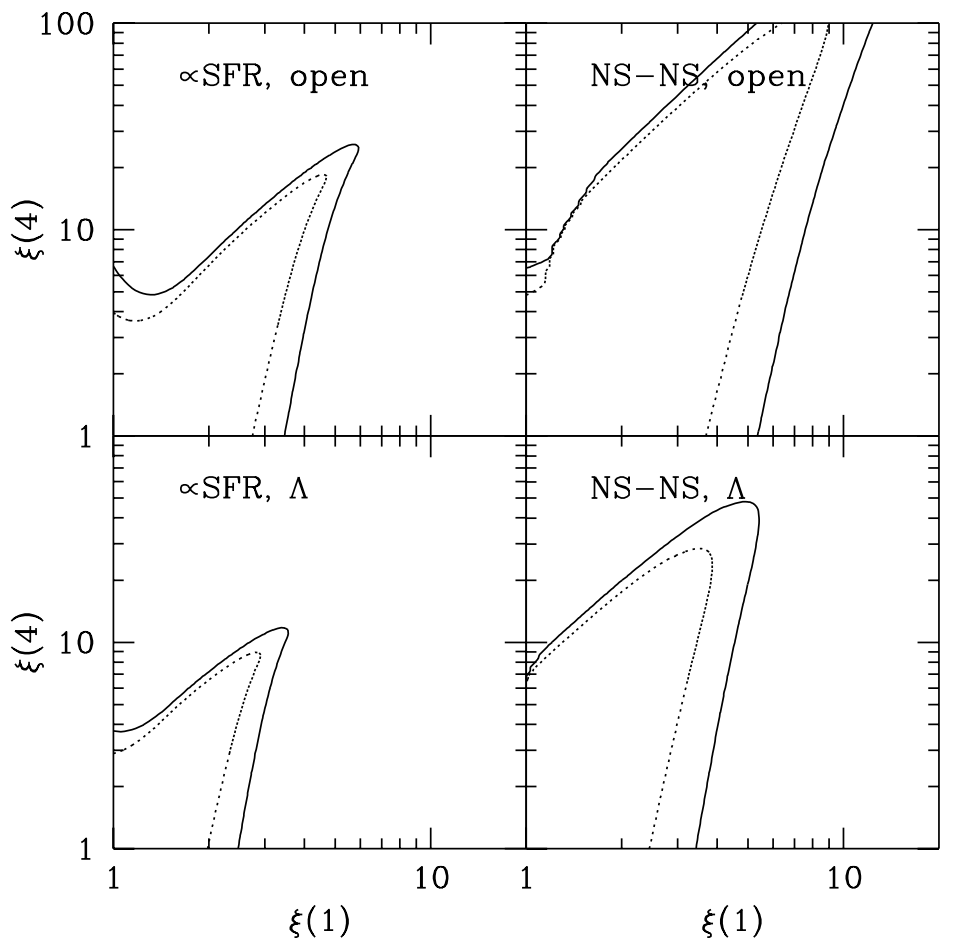

Fig. 13. - The same as Fig. 12, but only for the long GRBs with different cosmological parameters: $\left(h, \Omega_{0}, \Omega_{\Lambda}\right)=(0.6,0.2,0)$ and $(0.7,0.2,0.8)$ for the upper and lower panels, respectively. 


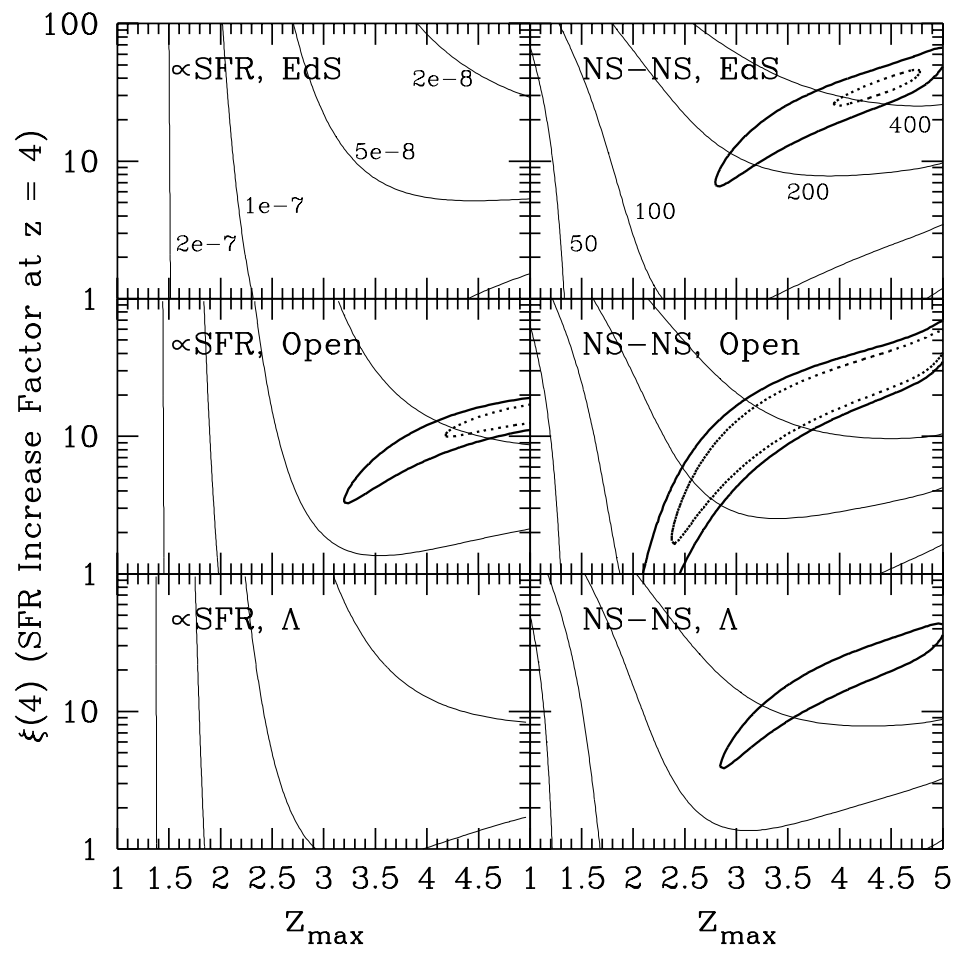

Fig. 14.- Allowed regions in $z_{\max }-\xi(4)$ plane are shown, assuming $\xi(1)=4$. The left panels are for the proportional model and the right for the NS-NS model, with the three different sets of cosmological parameters for each, as shown in the figure. The thick-solid line is 95 $\%$ C.L. constraint and the thick-dotted line for $68 \%$ C.L. In the upper-left and lower-left panels, there is no allowed region. The thin solid lines are contours of the normalization factor: production rate of GRBs per unit mass of star formation [GRB $M_{\odot}^{-1}$ ] in the left panels and beaming factor $(4 \pi / \Delta \Omega)$ in the right panels. 


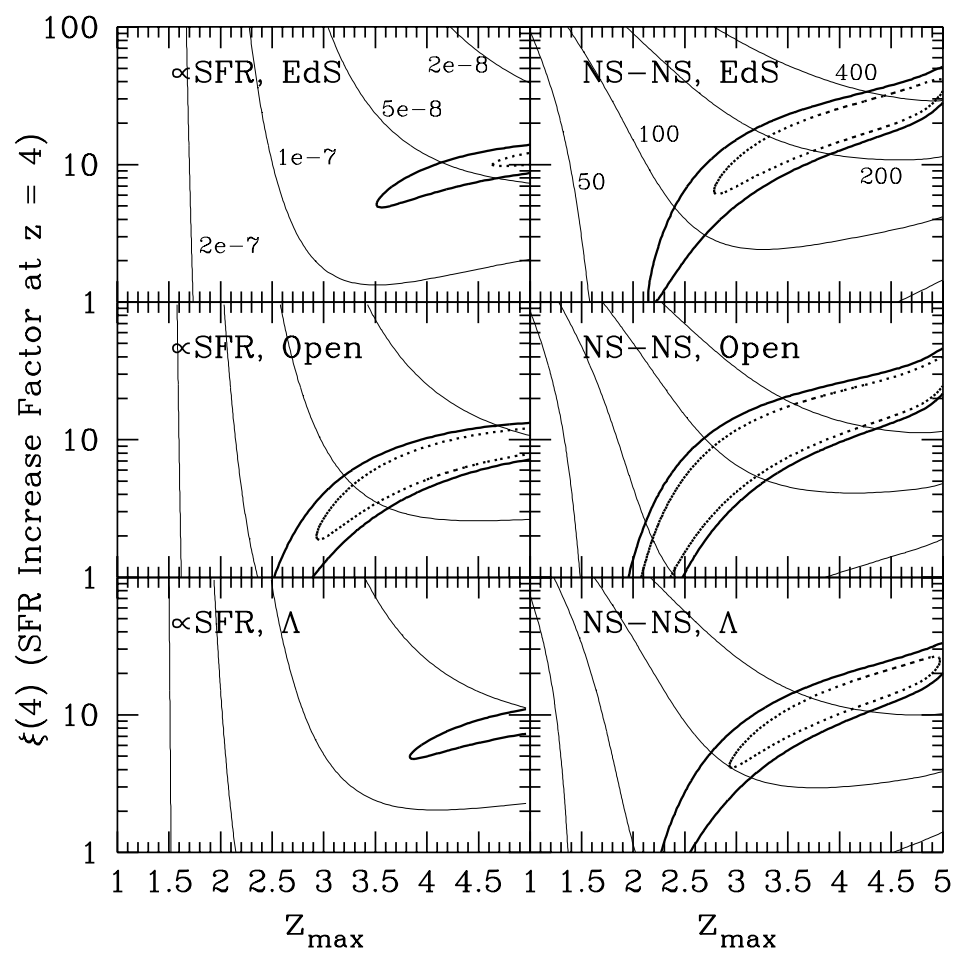

Fig. 15. - The same as Fig. 14, but $\xi(1)=3$. 


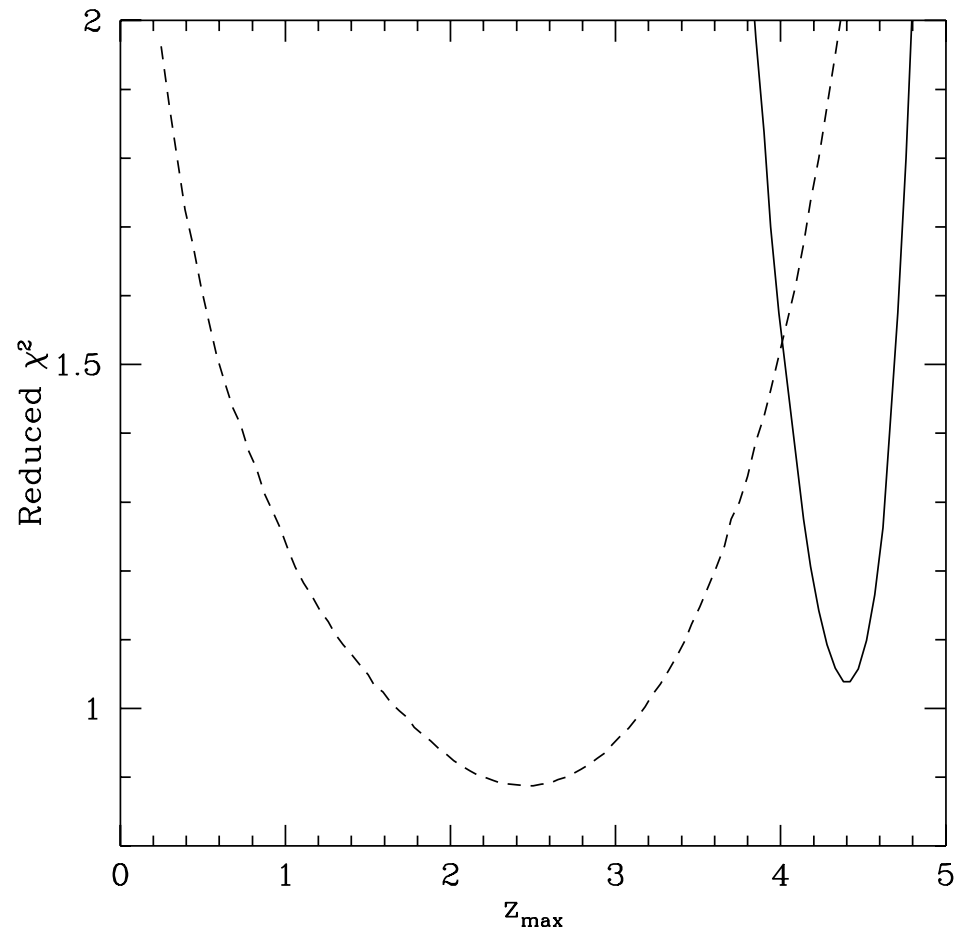

Fig. 16. - The reduced $\chi^{2}$ of the fit to the BATSE data as a function of $z_{\max }$, with a fixed cosmic SFR evolution $[(\xi(1), \xi(4))=(4,34.3)]$ in the NS-NS model. The solid line is for the long GRBs and the dashed line for the short GRBs. The Einstein-de Sitter universe with $h=0.5$ is assumed. 